\title{
New tick-borne encephalitis virus hot spot in Northern Zealand, Denmark, October 2019
}

Charlotte N Agergaard ${ }^{1}$, Maiken W Rosenstierne ${ }^{1}$, René Bødker², Morten Rasmussen ${ }^{1}$, Peter H S Andersen ${ }^{3}$, Anders Fomsgaard ${ }^{1}$

1. Department of Virus and Microbiological Special Diagnostics, Statens Serum Institut, Copenhagen, Denmark

2. Department of Veterinary and Animal Sciences, University of Copenhagen, Copenhagen, Denmark

3. Department of Infectious Disease Epidemiology and Prevention, Statens Serum Institut, Copenhagen, Denmark

Correspondence: Anders Fomsgaard (afo@ssi.dk)

During summer 2019, three patients residing by Tisvilde Hegn, Denmark were hospitalised with tickborne encephalitis (TBE) after tick bites. A new TBE virus (TBEV) micro-focus was identified in tick nymphs collected around a playground in Tisvilde Hegn forest. Estimated TBEV prevalence was $8 \%$, higher than in endemic areas around Europe. Whole genome sequencing showed clustering to a TBEV strain from Norway. This is the second time TBEV is found in Ixodes ricinus outside Bornholm, Denmark.

Tick-borne encephalitis virus (TBEV), a member of the family Flaviviridae, genus flavivirus, causes tick-borne encephalitis (TBE). In Denmark, TBE is endemic only on the island Bornholm, with an incidence of 4 per 100,000 inhabitants per year $[1,2]$. Here we report three clinical cases of TBE in patients hospitalised within a month and all residing at the boundary of the same forest, Tisvilde Hegn, in Northern Zealand.

\section{Clinical cases and virology analysis}

\section{Case 1}

Early July 2019, a man in his late 50s, was hospitalised with meningoencephalitis. He lives in a house ca $2.2 \mathrm{~km}$ from the Tisvilde Hegn forest border where he sometimes walks, and noticed a tick bite perhaps from his own garden. He developed typical two-phased disease, with 5 days of fever and gastrointestinal symptoms followed by 2 days of recovery, before developing meningoencephalitis. Serum and cerebrospinal fluid (CSF) samples were analysed at Statens Serum Institute, Copenhagen, Denmark. Serum samples from the day of hospitalisation were positive for anti-TBEV IgM and IgG (Enzygnost ELISA, Siemens, Erlangen, Germany) [3]. CSF showed elevated leukocyte count ( $48 \times 10^{9} / \mathrm{L}$; norm: o cells/L), increased protein (0.9 $\mathrm{g} / \mathrm{L}$, norm: $0.15-0.50$ ) and was positive for anti-TBEV IgM and IgG (Table 1). It was negative in RT-qPCRs for TBEV and flavivirus.

\section{Case 2}

Late June 2019, a man in his late 6os developed fever, influenza-like symptoms and increasing fatigue. The patient lives in a house with a garden bordering the same forest as Case 1. He uses the forest recreationally and experiences daily tick bites. About 4 weeks later, at the end of July, he was hospitalised with symptoms of meningitis in terms of nausea, vomiting, headache, photophobia, and pain from the neck and the back. CSF was analysed at Statens Serum Institute, Copenhagen, Denmark and showed pleocytosis (mononuclear leukocytes of $70 \times 10^{9} / \mathrm{L}$; norm: o cells/L), elevated protein level (1.46 g/L, norm: 0.15-0-50) and positive antiTBEV IgM and IgG titres, and negative in RT-qPCRs for flavivirus and TBEV. Serum samples were positive for anti-TBEV IgM and IgG (Table 1).

\section{Case 3}

Late June 2019, a woman in her 30 s was hospitalised with meningoencephalitis manifesting as headache, nausea, fatigue and photophobia. She presented with fever and dehydration, and was in a poor general condition. Blood samples at admission had a low platelet and leukocyte count, and liver parameters were elevated. Ten days earlier, at her summer cottage $3 \mathrm{~km}$ from the same forest, she spent time at a playground in the eastern part of the forest, where she noticed a tick bite on her thigh. About 5 days later, she developed back pain and fever, followed by influenza-like symptoms, loose stools and increasing fatigue. She had a few days of recovery, before hospitalisation for a week with clinical signs of meningoencephalitis. Unfortunately, no lumbar puncture or tests for TBE were initially performed. About 1 month later, in August 2019, she still suffered from fatigue and quick excitability and was seen at a hospital outpatient clinic. The patient asked to be tested for TBE and serology was strongly positive for anti-TBEV IgM and IgG. A serum sample 1 month later was still IgM and IgG positive (Table 1). 
TBEV-specific IgG and IgM antibody detection in tick-borne encephalitis cases by time of sampling, Northern Zealand, Denmark, $2019(\mathrm{n}=3)$

\begin{tabular}{|c|c|c|c|c|}
\hline Case & Material & Day of sampling ${ }^{a}$ & $\begin{array}{l}\text { TBEV IgG } \\
(\mathrm{U} / \mathrm{ml})^{\mathrm{b}}\end{array}$ & $\begin{array}{l}\text { TBEV IgM } \\
(\text { Index) }\end{array}$ \\
\hline \multirow{3}{*}{1} & Serum & 0 & 28.4 & 10.0 \\
\hline & CSF & 5 & 29.1 & 3.2 \\
\hline & Serum & 10 & 22.8 & 8.2 \\
\hline \multirow{4}{*}{2} & CSF & 0 & 43.7 & 1.1 \\
\hline & Serum & 0 & 106.8 & 6.9 \\
\hline & Serum & 2 & 118.5 & 6.6 \\
\hline & Serum & 10 & $73 \cdot 5$ & 5.8 \\
\hline \multirow{2}{*}{3} & Serum & 42 & 118.5 & 3.2 \\
\hline & Serum & 74 & 154.0 & 1.8 \\
\hline
\end{tabular}

CSF: cerebrospinal fluid; TBEV: tick-borne encephalitis virus.

${ }^{a}$ Day $0=$ day of hospital admission.

b The IgG antibody concentrations ( $\mathrm{U} / \mathrm{ml}$ ) were interpreted according to the manufacturer's instructions (range 7-70o $\mathrm{U} / \mathrm{ml}$ ).

'The specific IgM antibody concentrations are presented as indices according to manufacturer's instructions (index $1.0-1.4$ weakly positive and index $>1.4$ positive).

Field sampling and whole genome sequencing of tick-borne encephalitis virus

Ticks were collected by flagging, i.e. dragging of a $1 \times 1$ $\mathrm{m}$ white cloth through the grass, at Tisvilde Hegn in September and October 2019. The initial flagging took place at five different neighbouring sites, site $1-5$, in a part of the forest bordering the forest playground on the eastern side where Case 3 received a tick bite (Figure 1).

As of 26 September, a total of 725 ticks were collected, 626 nymphs and 99 adults, and divided into 24 pools (Table 2).

RNA was extracted using MagNA Pure Large Volume kit on a MagNA Pure 96 instrument (Roche Diagnostics, Risch-Rotkreuz, Switzerland), and a TBEV-specific RT-qPCR $[4,5]$ was run in a quality-controlled routine diagnostic reference laboratory. TBEV prevalence in individual ticks were estimated from the pooled samples using an online calculator for variable pool sizes while assuming a perfect diagnostic test [6].

Three pools containing nymphs from site 3 , bordering the playground, were all positive and of these, two pools were strongly positive (ct values: 17 and 20). Furthermore, one pool containing nymphs from site 5 , ca 50-100 $\mathrm{m}$ from the playground, was also positive (ct value: 35 ) (Table 2).

To further localise the TBEV micro-focus, the areas directly bordering the forest playground were divided into three smaller subsites and flagged once more in October 2019 (Figure 1). A total of 368 ticks, 348 nymphs and 20 adults, were collected and divided into 41 pools (Table 2). Four of 11 pools from site 3 A, two of 19 pools from site $3 B$ and all five pools from site $4 \mathrm{~A}$ contained nymphs positive for TBEV. No TBEV was found in pools of adult ticks. Sites $3 \mathrm{~A}$ and $4 \mathrm{~A}$, the two sites forming a $20 \mathrm{~m}$ wide belt along the eastern side of the playground were strongly positive (ct values: 15 and 18 ), as compared with the more distant site $3 B$. The joint prevalence of TBEV in sites $3 \mathrm{~A}$ and $4 \mathrm{~A}$ was estimated to $8 \%$ ( $95 \%$ confidence interval (Cl): 4-14\%) (Table 2).

Metagenomic whole genome sequencing of nine of the positive tick pools were performed using the Nextera XT DNA Library Prep Kit (Illumina Inc., San Diego, United States) and the Illumina MiSeq platform. For sequence comparison, the TBEV PCR-positive tick pool from Tokkekøb Hegn in 2009 was also full-genome sequenced and included in the analysis. Four complete whole genome sequences, three from Tisvilde Hegn and one from Tokkekøb Hegn, with an average coverage $100 x$ was obtained. Phylogenetic and molecular evolutionary analyses using MEGA X [7] of the fulllength genome sequences from Tisvilde Hegn showed that all three were identical and grouped closely with a TBEV strain from Mandal, Norway (Figure 2). In contrast, the TBEV sequence from Tokkekøb Hegn grouped with TBEV strains from Sweden (Figure 2). The sequences have been deposited in GenBank (accession numbers: MN735988, MN735989, MN735990 and MN735991*).

\section{Discussion}

The incidence of TBE has been increasing in Denmark, in its neighbouring countries as well and in the rest of Europe in recent years, which mirrors the increased abundance of ticks, the increased geographic spread and potentially climate changes [8-11]. The vector for the European virus subtype, TBEV-Eu, is Ixodes ricinus, which is prevalent in most of Europe and the dominant 


\section{FIGURE 1}

Map of Denmark, Northern Zealand and Tisvilde Hegn, 2019

A.

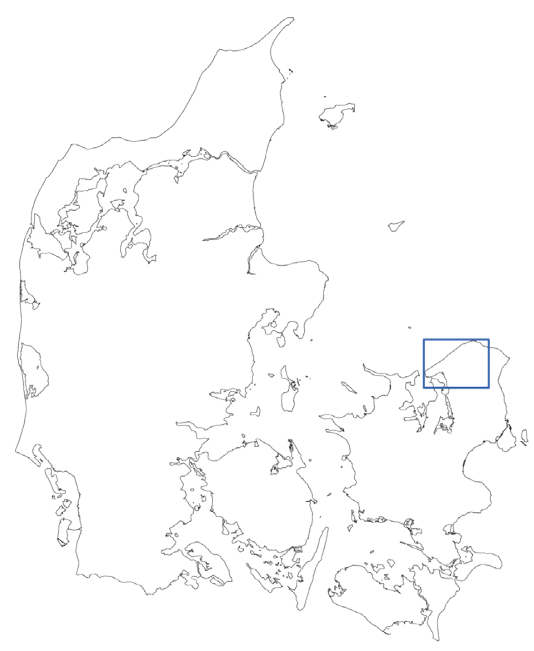

B.

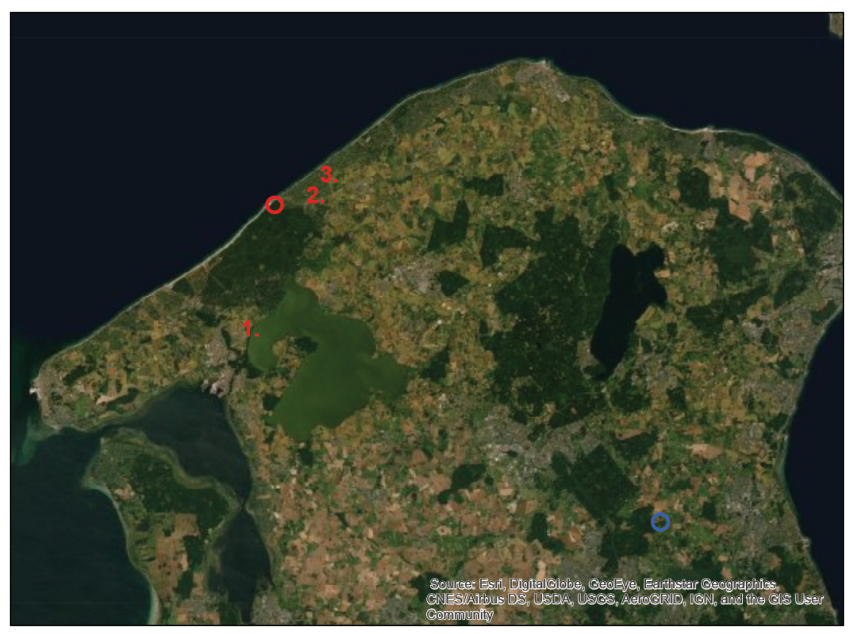

C.

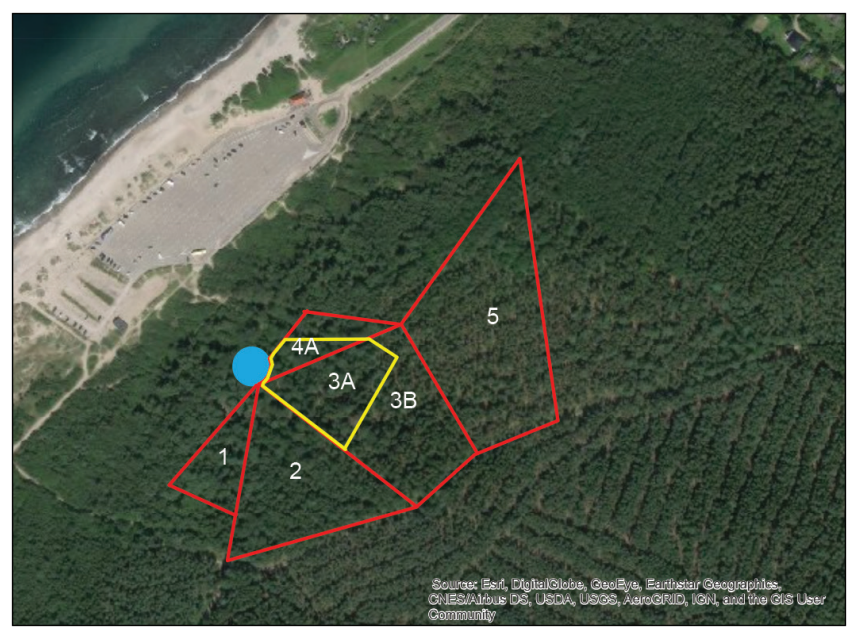

A. shows a map of Denmark and B. shows a map of Northern Zealand with the new tick-borne encephalitis (TBE) micro-focus in Tisvilde Hegn (red ring). It also shows a previous TBE micro-focus at Tokkekøb Hegn (blue ring) and the residence of the three human cases (in red). C. illustrates the tick flagging sites, the forest playground, marked with a blue dot, and the five initial flagging areas, sites 1-5. Areas 3 and 4 were divided into two new areas, of which 3A, 3B and 4A were flagged separately. Map source: Esri, DigitalGlobe, GeoEye, Earthstar, Geographics, CNES, Airbus DS, USDA, AeroGRID, IGN, and the GIS User Community. 
TABLE 2

TBEV-specific RT-qPCR and estimated virus prevalence, Northern Zealand, Denmark, 2019

\begin{tabular}{|c|c|c|c|c|c|c|c|c|}
\hline Sample site & $\underset{(2019)}{\text { Sampling date }}$ & Tick stage & $\begin{array}{l}\text { Number of } \\
\text { ticks }\end{array}$ & $\begin{array}{l}\text { Number of } \\
\text { pools }\end{array}$ & $\begin{array}{c}\text { Pool size } \\
\text { (Number of ticks) }\end{array}$ & $\begin{array}{c}\text { TBEV } \\
\text { RT-qPCR }\end{array}$ & $\begin{array}{l}\text { RT- } q P C R \\
(c t)\end{array}$ & $\begin{array}{c}\text { Estimated } \\
\text { prevalence (\%) } \\
(\mathrm{Cl} 95 \%)\end{array}$ \\
\hline \multirow{2}{*}{1} & \multirow{2}{*}{19 September } & Nymphs & 124 & 3 & $40,41,43$ & $0 / 3$ & No ct & \multirow{2}{*}{ All negative } \\
\hline & & Adults & 26 & 2 & $18^{\mathrm{a}}, 8^{\mathrm{b}}$ & $0 / 2$ & No ct & \\
\hline \multirow{2}{*}{2} & \multirow{2}{*}{19 September } & Nymphs & 91 & 2 & 45,46 & $0 / 2$ & No ct & \multirow{2}{*}{ All negative } \\
\hline & & Adults & 15 & 2 & $4^{\mathrm{a}}, 11^{\mathrm{b}}$ & $0 / 2$ & No ct & \\
\hline \multirow{2}{*}{3} & \multirow{2}{*}{26 September } & Nymphs & 216 & 5 & $\begin{array}{c}40,41,44,45 \\
46\end{array}$ & $3 / 5$ & $17,20,35$ & \multirow{2}{*}{$\begin{array}{c}2.0 \\
(1.0-6.0)\end{array}$} \\
\hline & & Adults & 33 & 2 & $18^{\mathrm{a}}, 15^{\mathrm{b}}$ & $0 / 2$ & No ct & \\
\hline \multirow{2}{*}{4} & \multirow{2}{*}{26 September } & Nymphs & 23 & 1 & 23 & $0 / 1$ & No ct & \multirow{2}{*}{ All negative } \\
\hline & & Adults & 2 & 1 & $1^{\mathrm{a}}$ & $0 / 1$ & No ct & \\
\hline \multirow[b]{2}{*}{5} & \multirow{2}{*}{26 September } & Nymphs & 172 & 4 & $41,41,43,47$ & $1 / 4$ & 35 & \multirow{2}{*}{$\begin{array}{c}1.0 \\
(0.0-29.0)\end{array}$} \\
\hline & & Adults & 23 & 2 & $11^{\mathrm{a}}, 12^{\mathrm{b}}$ & $0 / 2$ & No ct & \\
\hline \multirow[t]{2}{*}{$3 \mathrm{~A}$} & \multirow[t]{2}{*}{4 October } & Nymphs & 112 & 11 & $10^{c}, 12$ & $4 / 11$ & $\begin{array}{c}16,16,31 \\
34\end{array}$ & \multirow{2}{*}{$\begin{array}{c}4.0 \\
(1.0-10.0)\end{array}$} \\
\hline & & Adults & 4 & 2 & $1^{a}, 3^{b}$ & $0 / 2$ & No ct & \\
\hline \multirow{2}{*}{ 3B } & \multirow{2}{*}{4 October } & Nymphs & 192 & 19 & $10^{c}, 12$ & $2 / 19$ & 34,39 & \multirow{2}{*}{$\begin{array}{c}1.0 \\
(0.0-3.0)\end{array}$} \\
\hline & & Adults & 15 & 3 & $4^{\mathrm{a}}, 5^{\mathrm{b}}, 6^{\mathrm{b}}$ & $0 / 3$ & No ct & \\
\hline \multirow{2}{*}{$4 \mathrm{~A}$} & \multirow{2}{*}{4 October } & Nymphs & 44 & 5 & $10^{c}, 4$ & $5 / 5$ & $\begin{array}{c}15,18,31 \\
32,35\end{array}$ & \multirow[t]{2}{*}{ All positive } \\
\hline & & Adults & 1 & 1 & $1^{\mathrm{a}}$ & $0 / 1$ & No ct & \\
\hline $\begin{array}{l}3 \mathrm{~A} \text { and } 4 \mathrm{~A} \\
\text { jointly }\end{array}$ & 4 October & Nymphs & 156 & 16 & $10^{c}, 12,4$ & $9 / 16$ & NA & $\begin{array}{c}8.0 \\
(4.0-14.0) \\
\end{array}$ \\
\hline
\end{tabular}

$\mathrm{Cl}$ : confidence interval; ct: cycle threshold; NA: not applicable; TBEV: tick-borne encephalitis virus.

a Adult female ticks.

${ }^{\mathrm{b}}$ Adult male ticks.

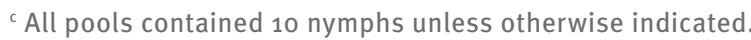

tick species in Denmark (>90\%) [12]. In 2009, two clinical cases of TBE were reported outside Bornholm and TBEV was detected in Northern Zealand in ticks collected in the forest of Tokkekøb Hegn, which is $40 \mathrm{~km}$ south-east of Tisvilde Hegn, in 2009, 2010 and 2011 $[4,5]$. Surprisingly, TBEV was no longer detected in the same area in Tokkekøb Hegn during 2016 and 2017 [13]. In 2018, another two human cases of TBE outside Bornholm were identified on the Island of Funen and in Jutland, respectively, but no new micro foci of TBEV has been localized [14], (data not shown).

All three patients presented here live close to Tisvilde Hegn in Northern Zealand, and had typical biphasic disease starting with fever, gastro-intestinal or influenza-like symptoms and fatigue, followed by a few days of recovery before clinical meningitis/meningoencephalitis at hospitalisation and neurologic sequelae in terms of primarily fatigue and dizziness.

Subsequent collection of $I$. ricinus ticks from a part of Tisvilde Hegn surrounding a well-visited forest playground, where Case 3 recalled a tick bite, identified a specific area adjacent to the playground to be an acute, new, high-risk TBEV micro-focus in Northern Zealand. The estimated high prevalence of TBEV is $8 \%$ at the centre of the focus which exceeds recent prevalence estimates of $0.6 \%$ from endemic Bornholm, as well as Denmark's neighbouring countries and most European countries $[4,5,8,10,11,13,15]$. The presence of the virus in nymphs, but not adult ticks, and the molecular evolutionary analyses of the homogeneous TBEV sequences suggests a single TBEV introduction in 2019, probably by migrating birds from Norway. Tisvilde Hegn and the forest playground is well-visited by Danish and international tourists, and containment measures such as fencing, grass cutting and signage along the playground's eastern side have been made in order to minimise the risk of further infections and spreading. 


\section{FIGURE 2}

Maximum-likelihood phylogenetic tree of TBEV full genome sequences, Northern Zealand, Denmark, 2019

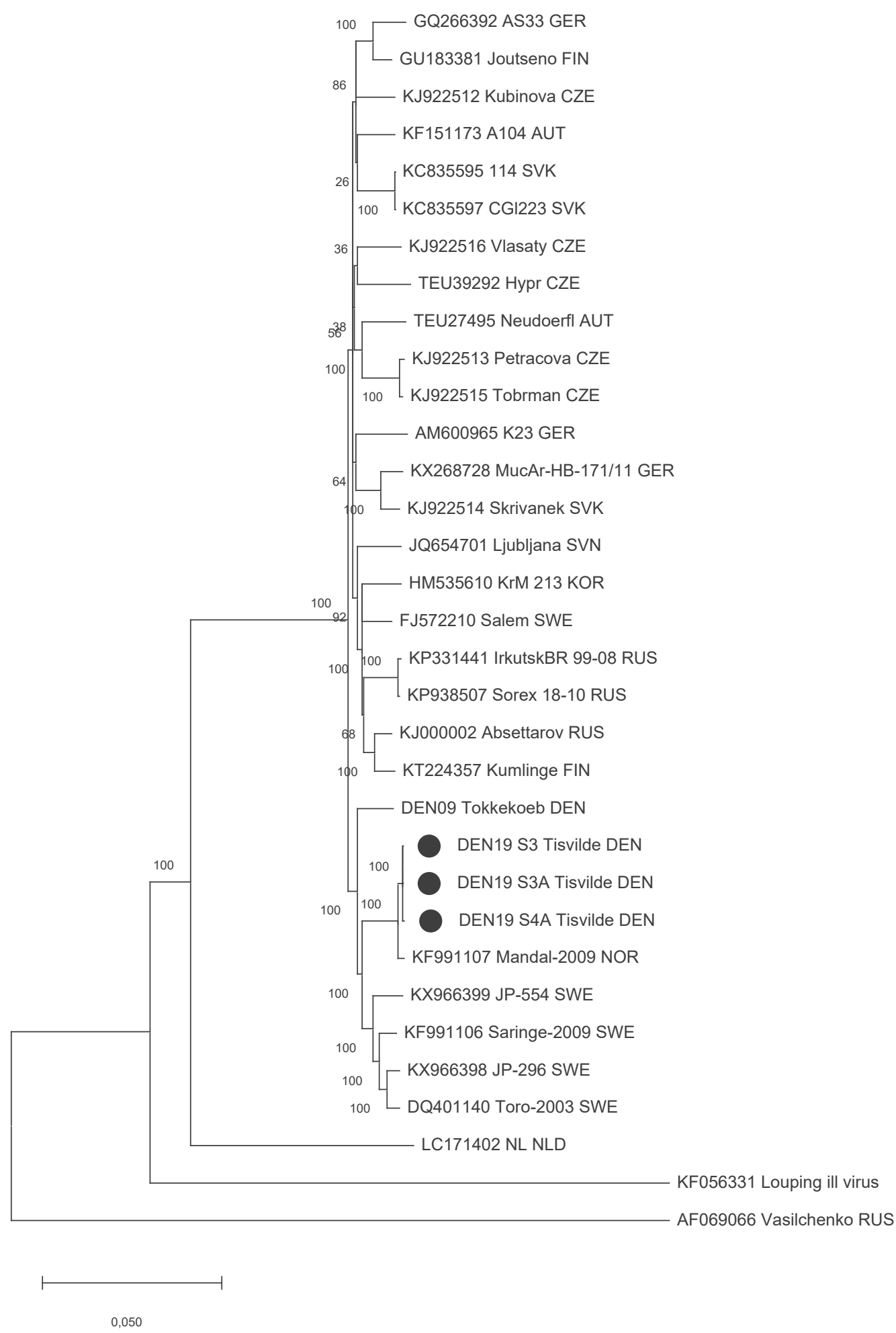

TBEV: tick-borne encephalitis virus.

Bootstrapping with 1,000 iterations was implemented for statistical support. The tree is drawn to scale, with branch lengths measured in the number of substitutions per site. The three full-length TBEV sequences obtained from Tisvilde Hegn are marked. The TBEV sequence from

Tokkekøb Hegn is shown, in addition to other TBEV full genome sequences from GenBank. 


\section{${ }^{*}$ Addendum}

The GenBank accession numbers were added on 4 December 2019.

\section{Acknowledgements}

We thank Susanne Lopez Rasmussen and Veronica Christensen for technical assistance and Lene Jung Kjær for generating the maps.

Funding statement: Collection of ticks were carried out by the National Vector Surveillance Program at University of Copenhagen funded by the Danish Veterinary and Food Administration.

\section{Conflict of interest}

None declared.

\section{Authors' contributions}

Charlotte N Agergaard: patient contact, clinical data collection, preparation of the manuscript.

Maiken W Rosenstierne: RT-qPCR analysis, revision of the manuscript.

René Bødker: flagging ticks, prevalence calculations, revision of the manuscript.

Morten Rasmussen: whole genome sequencing, NGS data analysis, revision of the manuscript.

Peter H. S. Andersen: epidemiological investigations, manuscript revision.

Anders Fomsgaard: patients/hospital contact, manuscript revision.

\section{References}

1. Laursen K, Knudsen JD. Tick-borne encephalitis: a retrospective study of clinical cases in Bornholm, Denmark. Scand J Infect Dis. 2003;35(5):354-7. https://doi. org/10.1080/00365540310005305 PMID: 12875531

2. Andersen NS, Larsen SL, Olesen CR, Stiasny K, Kolmos $\mathrm{HJ}$, Jensen PM, et al. Continued expansion of tick-borne pathogens: Tick-borne encephalitis virus complex and Anaplasma phagocytophilum in Denmark. Ticks Tick Borne Dis. 2019;10(1):115-23. https://doi.org/10.1016/j. ttbdis.2018.09.007 PMID: 30245088

3. Niedrig M, Avsic T, Aberle SW, Ferenczi E, Labuda M, Rozentale $B$, et al. Quality control assessment for the serological diagnosis of tick borne encephalitis virus infections. I Clin Virol. 2007;38(3):260-4. https://doi.org/10.1016/j. jcv.2006.12.013 PMID: 17267281

4. Fomsgaard A, Christiansen C, Bødker R. First identification of tick-borne encephalitis in Denmark outside of Bornholm, August 2009. Euro Surveill. 2009;14(36):19325. PMID: 19758543

5. Fomsgaard A, Fertner ME, Essbauer S, Nielsen AY, Frey S, Lindblom P, et al. Tick-borne encephalitis virus, Zealand, Denmark, 2011. Emerg Infect Dis. 2013;19(7):1171-3. https:// doi.org/10.3201/eid1907.130092 PMID: 23764123

6. Sergeant ESG. 2019. Epitools epidemiological calculators. Bruce ACT Australia: Ausvet Pty Ltd. [Accessed 13 Oct 2019]. Available from: http://epitools.ausvet.com.au

7. Kumar S, Stecher G, Li M, Knyaz C, Tamura K. MEGA X: Molecular Evolutionary Genetics Analysis across computing platforms. Mol Biol Evol. 2018;35(6):1547-9. https://doi. org/10.1093/molbev/msyo96 PMID: 29722887

8. European Centre for Disease Prevention and Control (ECDC). 2018. Tick-borne encephalitis. Annual Epidemiological report for 2017. Stockholm: ECDC; 2019. [Accessed 15 Oct 2019]. Available from: https://www.ecdc europa eu/sites/default/ files/documents/AER_for_2017-tick-borne-encephalitis_0.pdf

9. Lindquist L, Vapalahti O. Tick-borne encephalitis. Lancet. 2008;371(9627):1861-71. https://doi.org/10.1016/S01406736(08)60800-4 PMID: 18514730

10. Jaenson TGT, Hjertqvist $M$, Bergström T, Lundkvist $A$. Why is tick-borne encephalitis increasing? A review of the key factors causing the increasing incidence of human TBE in Sweden. Parasit Vectors. 2012;5(1):184. https://doi.org/10.1186/17563305-5-184 PMID: 22937961

11. Sidorenko M, Radzievskaja J, Rosef O, Paulauskas A. Investigation of the tick-borne encephalitis virus in Norway. Biologija (Vilnius). 2018;64(2):172-8. https://doi.org/10.6001/ biologija.v64i2.3741

12. Kjær LJ, Soleng A, Edgar KS, Lindstedt HEH, Paulsen KM, Andreassen AK, et al. A large-scale screening for the taiga tick, Ixodes persulcatus, and the meadow tick, Dermacento reticulatus, in southern Scandinavia, 2016. Parasit Vectors. 2019;12(1):338. https://doi.org/10.1186/s13071-019-3596-3 PMID: 31288866

13. Petersen A, Rosenstierne MW, Rasmussen M, Fuursted K, Nielsen HV, O'Brien Andersen L, et al. Field samplings of Ixodes ricinus ticks from a tick-borne encephalitis virus micro-focus in Northern Zealand, Denmark. Ticks Tick Borne Dis. 2019;10(5):1028-32. https://doi.org/10.1016/j. ttbdis.2019.05.005 PMID: 31151922

14. Laugesen NG, Stenør C. [Tick-borne encephalitis-associated meningoradiculoneuritis acquired in the south-western part of Denmark]. Ugeskr Laeger. 2019;181(27):Vo3190197. PMID: 31267941

15. Andersen NS, Bestehorn M, Chitimia-Dobler L, Kolmos HJ, Jensen PM, Dobler G, et al. Phylogenetic characterization of tick-borne encephalitis virus from Bornholm, Denmark. Ticks Tick Borne Dis. 2019;10(3):533-9. https://doi.org/10.1016/j. ttbdis.2018.12.008 PMID: 30704909

\section{License, supplementary material and copyright}

This is an open-access article distributed under the terms of the Creative Commons Attribution (CC BY 4.0) Licence. You may share and adapt the material, but must give appropriate credit to the source, provide a link to the licence and indicate if changes were made.

Any supplementary material referenced in the article can be found in the online version.

This article is copyright of the authors or their affiliated institutions, 2019. 